Original paper

\title{
Improving molecular radiotherapy dosimetry using anthropomorphic calibration
}

\author{
Emlyn Price ${ }^{\mathrm{a}, \mathrm{b}, *}$, Andrew P. Robinson ${ }^{\mathrm{a}, \mathrm{c}}$, David M. Cullen ${ }^{\mathrm{a}}$, Jill Tipping ${ }^{\mathrm{b}}$, Nick Calvert ${ }^{\mathrm{b}}$, \\ David Hamilton $^{\mathrm{b}}$, Christopher Oldfield ${ }^{\mathrm{a}}$, Emma Page ${ }^{\mathrm{b}, \mathrm{a}}$, Ben Pietras ${ }^{\mathrm{a}}$, Andrew Smith ${ }^{\mathrm{a}}$ \\ ${ }^{a}$ Schuster Laboratory, School of Physics and Astronomy, The University of Manchester, Oxford Road, Manchester M13 9PL, United Kingdom \\ ${ }^{\mathrm{b}}$ Christie Medical Physics and Engineering (CMPE), The Christie NHS Foundation Trust, Wilmslow Road, Manchester M20 4BX, United Kingdom \\ ${ }^{\mathrm{c}}$ National Physical Laboratory, Hampton Road, Teddington, Middlesex TW11 OLW, United Kingdom
}

\section{A R T I C L E I N F O}

\section{Keywords:}

Activity quantification

3D printing

MRT

Dosimetry

\begin{abstract}
A B S T R A C T
The optimised delivery of Molecular Radiotherapy requires individualised calculation of absorbed dose to both targeted lesions and neighbouring healthy tissue. To achieve this, accurate quantification of the activity distribution in the patient by external detection is vital.

Methods: This work extends specific anatomy-related calibration to true organ shapes. A set of patient-specific 3D printed organ inserts based on a diagnostic CT scan was produced, comprising the liver, spleen and both kidneys. The inserts were used to calculate patient-specific calibration factors for ${ }^{177} \mathrm{Lu}$. These calibration factors were compared with previously reported calibration factors for corresponding organ models based on the Cristy and Eckerman phantom series and for a comparably sized sphere. Monte Carlo calculations of the patientspecific radiation dose were performed for comparison with current clinical dosimetry methods for these data. Results: Patient-specific calibration factors are shown to be dependent on the volume, shape and position of the organ containing activity with a corresponding impact on the calculation of the dose to the patient. The impact of organ morphology on calculated dose is reduced when the dominant contributor to dose is beta particles. This is due to the small range of beta particles in tissue. Overestimations of recovered activity and hence dose of up to $135 \%$ are observed.

Conclusion: For accurate quantification to be performed calibration factors accounting for organ size, shape and position must be used. Such quantification is vital if accurate, patient-specific dosimetry is to be achieved.
\end{abstract}

\section{Introduction}

Molecular Radiotherapy (MRT) is growing in popularity as a combined therapy and imaging procedure. In current practice a standard activity is often administered. This results in a wide range of absorbed doses to organs of interest in different patients, as much as 14-32 Gy to the kidneys in ${ }^{177} \mathrm{Lu}$-Dotatate therapies [1].

The optimisation of MRT therapies requires individualised calculation of absorbed dose to both targeted lesions and organs at risk. Such patient-specific dosimetry is now regarded as an essential component of MRT therapies [2]. In a recent review of the use of dosimetry in the clinical practice of MRT it was concluded that 'evidence strongly implies a correlation between the absorbed doses delivered and the response and toxicity' and that 'dosimetry-based personalised treatments will improve outcomes and increase survival' [3].
A variety of tools for performing dosimetry clinically are available. These include OLINDA/EXM versions 1 and 2 which implement the MIRD schema for pre-defined mathematical models of organs, the Cristy and Eckerman (C\&E) models and the ICRP 89 phantom respectively $[4,5]$. Patient-specific dosimetry requires the measurement of the activity distribution in the patient. For the majority of MRT radiopharmaceuticals the activity distribution is measured using quantified SPECT imaging [6], or in the case of positron-emitting nuclides quantified PET imaging [7]. In order to quantify the activity in patients the imaging system must be calibrated to relate the detected count rate to activity. This is clinically performed using standard objects such as spheres [6]. Current SPECT quantification requires multiple interlinked corrections which may have varying performance throughout a typical clinical image $[6,8]$. It is therefore essential to have realistic test objects which can represent a clinical activity distribution with known activity

\footnotetext{
*Corresponding author at: Schuster Laboratory, School of Physics and Astronomy, The University of Manchester, Oxford Road, Manchester M13 9PL, United Kingdom.

E-mail address: emlyn.price@manchester.ac.uk (E. Price).
} 
concentrations to validate quantitative imaging (QI) [6].

Recently there has been increased interest in the application of additive manufacturing (3D printing) to the production of realistic phantoms, which can be filled with a radiopharmaceutical solution for SPECT and PET imaging. SPECT imaging with ${ }^{99 \mathrm{~m}} \mathrm{Tc}$ and PET imaging with ${ }^{18} \mathrm{~F}$ of a 3D printed phantom based on a patient CT image was first reported in [9]. The development of a selective internal radiation therapy (SIRT) specific liver phantom was recently reported [10]. Nuclide-dependent SPECT/CT calibration factors have been determined for a set of 3D printed kidney dosimetry phantoms and their spherical counterparts to assess the accuracy of quantitative imaging for internal renal dosimetry [11]. In related work we have recently made available a series of 3D printed phantom inserts based on the C\&E mathematical models [12]. These C\&E inserts were used to demonstrate that nonspherical SPECT calibration factors from 3D printed phantom inserts can significantly improve the accuracy of whole organ activity quantification for MRT [12].

In the present work a patient-specific phantom has been produced using 3D printed photopolymers. A diagnostic CT image, taken as part of a therapy regimen for a patient receiving MRT for neuroendocrine tumours (NETs), was used to design the geometry of the manufactured phantom. Patient-specific calibration factors for SPECT activity quantification of ${ }^{177} \mathrm{Lu}$ are reported and compared to previously reported experimental calibration factors (based on a $113 \mathrm{ml}$ sphere and the C\&E models [12]). The impact of patient-specific organ geometry on SPECT calibration factors is discussed. Absorbed dose calculations using the OLINDA/EXM 1 package [4] for patient representative activity distributions, calculated using the sphere, organ-specific and patient-specific calibration factors, are compared. A patient-specific Monte Carlo simulation of dose distribution is used to provide a 'ground-truth' absorbed dose calculation. This information allows the accuracy of MRT dose calculations to be assessed, providing a benchmark for clinical accuracy. A potential link to individual clinical outcomes is also discussed.

\section{Materials and methods}

\subsection{Patient-specific phantom inserts}

Phantom inserts representing the kidneys, liver and spleen were produced using diagnostic CT images from a patient receiving MRT for neuroendocrine disease (Peptide Receptor Radionuclide Therapy, PRRT). The organs of interest were manually outlined to produce Volumes Of Interest (VOIs), subsequently smoothed and converted to STeroLithography (STL) format. This was used as an internal volume for a $2 \mathrm{~mm}$ shell except for the liver for which a $3 \mathrm{~mm}$ shell was used due to its large dimensions. Integrated attachment and filling ports were added to the model for each organ allowing them to be mounted in phantoms. A custom made elliptical phantom (CATIE, the Christie Anatomical Tomographic Imaging Ellipse) was produced to allow the inserts to be mounted in their correct relative anatomical positions in a volume approximating a real patient geometry. The elliptical cylinder is $300 \mathrm{~mm}$ tall internally with major and minor axes of $360 \mathrm{~mm}$ and $320 \mathrm{~mm}$ respectively. Fig. 1 shows (a) the segmented CT, (b) the final CAD design for the organ inserts and elliptical phantom and (c) the completed phantom assembly.

The phantom inserts were printed in an acrylic-based photopolymer using a commercial 3D printing service. Table 1 shows the volumes of the 3D printed inserts in comparison to the initial VOIs. For all printed inserts the mean difference in volume from the original CT VOIs was 2.6 CT voxels, less than $5 \mathrm{ml}$.

\subsection{Detector calibration}

A calibration factor, cf, can be defined for a complete reconstructed SPECT image or a specific VOI on the image which relates the count rate (counts per second, cps) in that region to total activity (MBq) to provide activity quantification of SPECT imaging,

$\mathrm{cf}=\frac{\text { counts }}{\text { total activity } \cdot \text { scan duration }}$.

The calibration factor is specific to the camera, collimator, reconstruction parameters and applied image corrections. Spill out of counts from a VOI due to the finite spatial resolution of the SPECT camera causes the measured calibration factor to deviate from the true camera sensitivity. The variation in calibration factor is volume dependent, accounting for partial volume effects [6], and a clear relationship between measured whole organ calibration factors and VOI shape has been demonstrated [12]. If a large enough volume is used, in order to minimise partial volume effects, the true camera sensitivity, $\mathrm{cf}_{\text {true }}$, can be measured. The value of $\mathrm{cf}_{\text {true }}(11.74 \pm 0.02 \mathrm{cps} / \mathrm{MBq})$ for the scanner used in this work was previously determined in reference [12] by imaging a 7.31 cylindrical phantom containing a uniform distribution of ${ }^{177} \mathrm{Lu}$ solution. Following system performance QC to ensure consistency of system operation this value was used in this work to allow direct comparison with results from reference [12].

As has been shown in reference [12], it is possible to calculate the ratio of the calibration factor for a VOI to $\mathrm{cf}_{\text {true }}$ using the shape of the VOI. The ratio is given by

$\frac{\mathrm{cf}_{\mathrm{voi}}}{\mathrm{cf}_{\text {true }}}=1-\frac{\sum_{i=i}^{\mathrm{nVoxels}} \alpha_{i}}{\text { nVoxels }}$,

where for an individual voxel centred at $x_{i}, y_{i}, z_{i}$ with $x_{a}, x_{b}$, $y_{a}, y_{b}, z_{a}, z_{b}$ being the perpendicular distances to the closest VOI boundary,

$\alpha_{i}=1-\int_{z_{a}-z_{i}}^{z_{b}-z_{i}} \int_{y_{a}-y_{i}}^{y_{b}-y_{i}} \int_{x_{a}-x_{i}}^{x_{b}-x_{i}} g\left(x_{i}, y_{i}, z_{i}, \sigma\right) d x d y d z$.

$g\left(x_{i}, y_{i}, z_{i}, \sigma\right)$ is defined as

$g\left(x_{i}, y_{i}, z_{i} \sigma\right)=\frac{1}{\sigma^{3}(2 \pi)^{\frac{2}{3}}} \exp \left(-\left(\frac{x^{2}+y^{2}+z^{2}}{2 \sigma^{2}}\right)\right)$

and

$\sigma(\mathrm{FWHM})=\frac{\mathrm{FWHM}}{2 \sqrt{2 \ln 2}}$,

where FWHM is the full width at half maximum of the SPECT camera. The theoretical values of $\frac{\mathrm{cf}_{\mathrm{voi}}}{\mathrm{cf} \mathrm{f}_{\text {true }}}$ are compared with experiment for images reconstructed using attenuation correction only to isolate the impact of organ shape. Theoretical calibration factors were also calculated for spheres of the same volume (subject to voxelisation effects) as each insert.

\subsection{SPECT phantom data}

For all the scans undertaken in this work the phantom inserts were filled with ${ }^{177} \mathrm{Lu}$-DотAтATE in $\mathrm{NaCl}$ solution, using patient-representative activities as shown in Table 2. Each insert was scanned individually using an Infinia-Hawkeye-4 SPECT/CT camera (GE Healthcare) to calculate an organ specific calibration factor (termed 'Calibration'). For these initial calibration scans each insert was positioned centrally in a water-filled elliptical Jaszczak phantom, where possible. However the liver insert is too large to fit centrally in the Jaszczak phantom so was scanned in the anatomical position in the CATIE phantom, which was also filled with water. The spleen insert was subsequently imaged alone in the anatomical position to examine the effect of radial position on calibration factor. The inserts were also fully assembled in the CATIE phantom (as shown in Fig. 1)) and scanned with background activity in the phantom body to provide a clinically representative activity distribution. The SPECT/CT data were acquired using the parameters corresponding to a clinical ${ }^{177}$ Lu-Dotatate post-therapy scan (Table 3). 

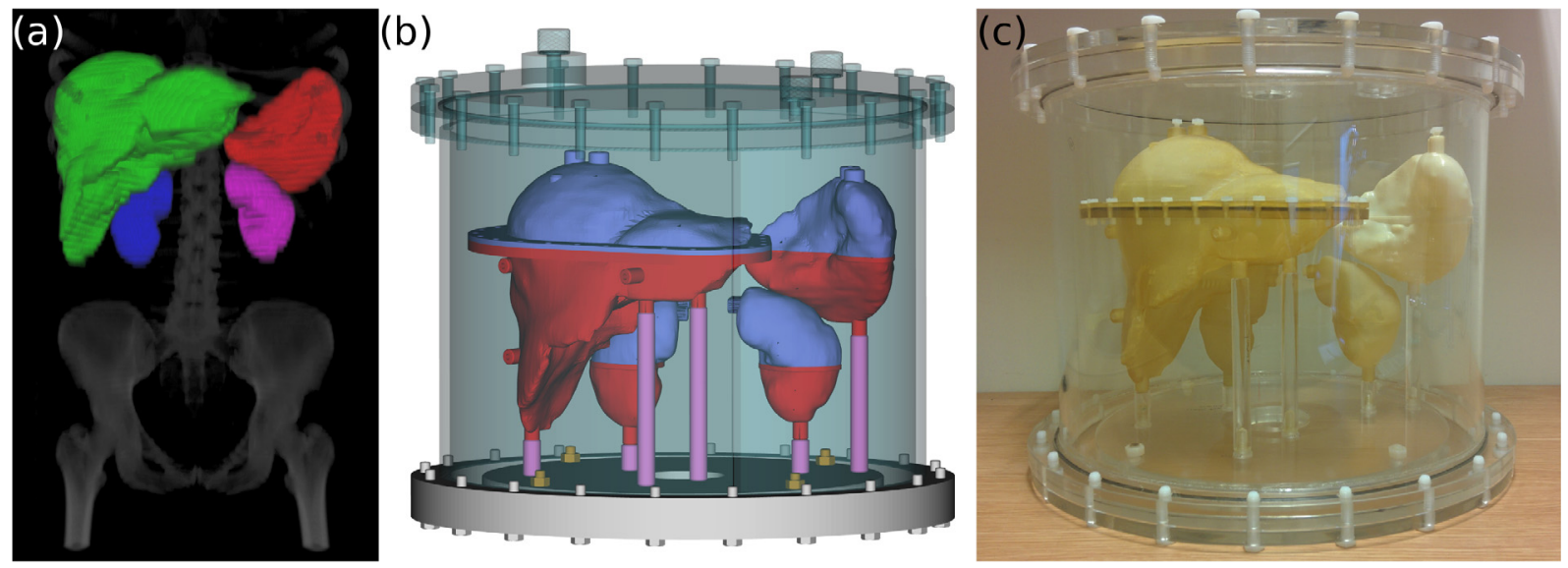

Fig. 1. (a) Segmented organ VOIs displayed on patient CT data. (b) Model of organ inserts in elliptical phantom with correct relative anatomical positions. (c) Assembled 3D printed organ inserts.

Table 1

Volumes of organs as defined on the patient CT and corresponding 3D printed phantom inserts. There is an uncertainty on the size of the VOI defined from the patient, but for the purposes of the phantom design the VOI was considered to be the 'true' patient. The volumes of the 3D printed C\&E inserts, taken from [12], are also shown.

\begin{tabular}{lrrr}
\hline & \multicolumn{3}{c}{ Volume (ml) } \\
\cline { 2 - 4 } Insert & CT VOI & 3D Printed & C\&E insert \\
\hline Liver & 1920.16 & $1914.9 \pm 0.20$ & $1577.49 \pm 0.02$ \\
Spleen & 400.38 & $390.66 \pm 0.02$ & $195.26 \pm 0.02$ \\
Left kidney & 169.45 & $167.72 \pm 0.02$ & $169.81 \pm 0.02$ \\
Right kidney & 157.39 & $154.81 \pm 0.02$ & $169.81 \pm 0.02$ \\
\hline
\end{tabular}

The phantom was scanned, for each insert, in the same central position on the scanner bed with a fixed head rotation radius of $25 \mathrm{~cm}$ to ensure a constant SPECT resolution. Each scan was performed once. A fixed rotation radius was used to allow direct comparison between the scans and simulation. Clinical quality control (QC) is carried out daily on the SPECT scanner to ensure consistent performance. The activity in each insert was calculated using measurements taken using a Capintec radionuclide calibrator which is traceable to a primary standard. The accuracy of the radionuclide calibrator is certified to be within $2 \%$ of the primary standard for ${ }^{177} \mathrm{Lu}$.

Projection images of the phantom were acquired using the ${ }^{177} \mathrm{Lu}$ $208 \mathrm{keV}$ photopeak, along with adjacent scatter windows to allow a triple energy window scatter correction to be applied [8]. The $208 \mathrm{keV}$ photopeak was chosen, rather than the $113 \mathrm{keV}$ photopeak, to reduce the effect of scattered photons on the calibration [13]. All projections were acquired using medium energy general purpose (MEGP) collimators. Dose-saving hybrid CT scans were acquired to allow attenuation correction and accurate delineation of the phantom insert boundaries in accordance with clinical protocol.
Table 3

SPECT tomographic acquisition parameters corresponding to clinical ${ }^{177} \mathrm{Lu}-$ DotatATE post-therapy scan.

\begin{tabular}{ll} 
Pixels & $128 \times 128$ \\
Number of views per head & $30(60$ in total) \\
Time per view & $40 \mathrm{~s}$ \\
Rotation radius & $25 \mathrm{~cm}$ \\
Photopeak window & $208 \mathrm{keV} \pm 10 \%$ \\
Scatter windows & $181 \mathrm{keV} \pm 3 \%, 236 \mathrm{keV} \pm 3 \%$ \\
\hline
\end{tabular}

The phantom data were reconstructed using a standard clinical Ordered Subsets Expectation Maximisation (OSEM) algorithm used for dosimetry (GE OSEM) with 4 iterations, 10 subsets and no resolution recovery or post filter applied. The images were reconstructed with CTderived attenuation correction and triple energy window scatter correction. Fig. 2 shows a comparison of reconstructed SPECT images of (a) patient data and (b) the corresponding phantom data.

\subsection{Activity quantification}

Calibration factors for each 3D printed insert were calculated by outlining the insert on the CT scans, transferring the resultant VOI to the co-registered SPECT image and recording the total number of counts in the VOI. The uncertainties on the VOI counts were calculated using the 'random' method of boundary perturbation described in [14], where the boundary of each VOI was shifted and the variation in counts observed for twenty different perturbations. The organ-specific calibration factors, including those for the spleen in both positions, were then used to quantify the activity (Eq. (6)) in each insert in the SPECT scans of the full phantom. Calibration factors for the corresponding C\&E organs and a $113 \mathrm{ml}$ sphere, calculated in [12], were also used to quantify the activity in the patient-specific inserts in order to assess the impact of calibration factor on the recovered activity and calculated dose. The $113 \mathrm{ml}$ sphere was used as it is large enough that the impact

Table 2

Patient-specific insert activity $\left({ }^{177} \mathrm{Lu}\right)$ for calibration scans (Calibration) and full phantom scans with (BG) and without (No BG) background activity in the CATIE phantom body.

\begin{tabular}{|c|c|c|c|c|c|}
\hline \multirow[t]{2}{*}{ Insert } & \multicolumn{2}{|c|}{ Activity (MBq) } & \multicolumn{2}{|c|}{ Activity concentration $(\mathrm{MBq} / \mathrm{ml})$} & \multirow{2}{*}{$\begin{array}{l}\text { Insert to BG ratio } \\
\text { (concentration) }\end{array}$} \\
\hline & Calibration & Full Phantom & Calibration & Full Phantom & \\
\hline Liver & $389 \pm 8$ & $348 \pm 7$ & $0.203 \pm 0.004$ & $0.182 \pm 0.004$ & $5.08 \pm 0.04$ \\
\hline Spleen & $842 \pm 17$ & $121 \pm 3$ & $0.750 \pm 0.016$ & $0.309 \pm 0.007$ & $8.61 \pm 0.12$ \\
\hline Left kidney & $414 \pm 9$ & $128 \pm 3$ & $2.47 \pm 0.05$ & $0.761 \pm 0.018$ & $21.22 \pm 0.30$ \\
\hline Right kidney & $388 \pm 8$ & $120 \pm 3$ & $2.50 \pm 0.05$ & $0.773 \pm 0.020$ & $21.5 \pm 0.4$ \\
\hline Background & $\mathrm{N} / \mathrm{A}$ & $813 \pm 16$ & N/A & $0.0358 \pm 0.0007$ & N/A \\
\hline
\end{tabular}



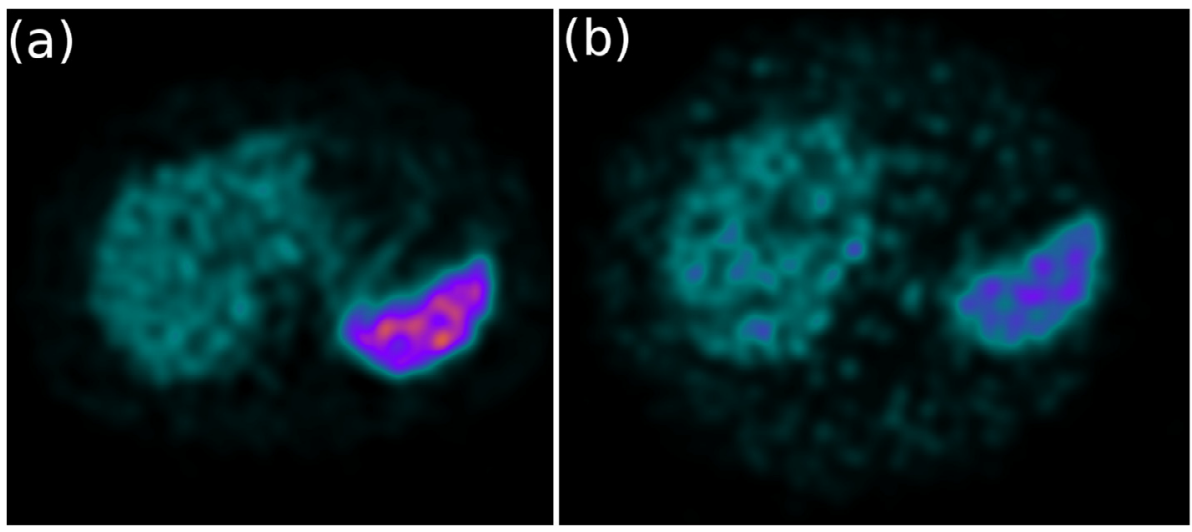

Fig. 2. A comparison of equivalent slices of reconstructed SPECT scans of (a) patient data and (b) corresponding patient-specific 3D printed inserts inside the phantom. The spleen insert contains a lower activity concentration than the patient data. The patient also has uptake in organs and tumours which are not included in the phantom.

of partial volume effects will be reduced.

The activity calculated in an insert, $A_{\text {quantified, }}$ is given by

$A_{\text {quantified }}=\frac{\text { counts }}{\text { cf.scan duration }}$

For a phantom with known activities in the inserts a recovery factor, $\mathrm{RF}$, can defined as,

$\mathrm{RF}=1-\frac{A_{\text {administered }}-A_{\text {quantified }}}{A_{\text {administered }}}$,

where $A_{\text {administered }}$ is the known administered activity in an insert. A recovery factor less than 1.0 corresponds to an underestimation of the quantified activity in the insert and values greater than 1.0 represent an overestimation of the administered activity.

\subsection{Dosimetry calculations}

\subsubsection{MIRD absorbed dose calculations}

The quantified activities in the anatomically assembled organs, determined using different calibration factors, were used to calculate the doses to each organ insert using the OLINDA/EXM package [4]. The dose to each organ was also calculated using the known activity in each insert to provide a calculation independent of the SPECT measurements. OLINDA/EXM implements the MIRD schema [15] to calculate the doses to organs using pre-calculated S-factors. S-factors are provided in OLINDA/EXM for a large number of radionuclides and specific phantom models. These are used to report the dose to organs for a given distribution of activity between organs, assuming uniform uptake in individual organs [4]. The dose to each organ from the activity it contains (the self-dose), and the dose from each organ to the others (the cross-dose) is reported. The kidneys are considered to be a single organ in the C\&E phantom. The masses of the organs were calculated from the volumes of the VOIs which define the inserts and used to scale the masses in OLINDA/EXM. In this study there was no biological half-life to be considered and only the physical half-life was used to calculate the time-integrated activity coefficient as defined in [15].

\subsubsection{Monte Carlo simulated dose calculations}

Monte Carlo simulations of absorbed dose from ${ }^{177} \mathrm{Lu}$ were undertaken using GATE v8.0 [16], an extension to GEANT4 [17] for medical tomography. The CT VOIs on which the 3D printed inserts were based were used to define the voxelised geometry of the simulation and sources of activity. The contents of the patient body and the organs were set to be homogeneous 'soft tissue' as defined in [18] and a homogeneous distribution of activity in each organ was assumed. A further VOI defining the patient torso was added to delineate the volume containing the organs. For all simulations the recommended physics model for this energy range in GATE, 'EM option 3', was used.

The absorbed dose was recorded in a voxelised 3D dose map with voxels the same size as those used to define the geometry and sources.
Both the gamma and beta radiation components of the dose were recorded. Self-dose and cross-dose components for each organ VOI were extracted from the dose map. The dose to the combined 'kidneys' organ as used in OLINDA/EXM was also calculated directly at this stage. The output of the patient-specific simulation was converted to doses from the administered activities and compared to the OLINDA/EXM dose calculations.

A simulation was also performed for validation using voxelised models of the C\&E kidneys, liver and spleen and the output compared to that of OLINDA/EXM. These models were also produced with voxels the same size as those in the clinical CT scan.

\section{Results}

\subsection{Patient-specific calibration factors}

Patient-specific calibration factors for the phantom inserts are given in Table 4. Significant differences can be seen between the calibration factors for the kidneys and those for the liver and spleen. This is due to the variation in size, shape and position of the inserts. The differences between the calibration factors for the spleen in the central position and anatomical position are only due to the location of the insert. A similar variation in calibration factor with position has been observed for spheres in [19]. The experimental and theoretical ratios (using a FWHM of $16 \mathrm{~mm}$ ) between $\mathrm{cf}_{\mathrm{voi}}$ and $\mathrm{cf}_{\text {true }}$ for each insert are shown in Fig. 3. Also shown are the ratios for spheres of the same volume as each insert. The differences between the theoretical ratios for the insert VOI and a sphere of corresponding volume are 5.0\% for the left kidney, $1.9 \%$ for the right kidney, $6.4 \%$ for the spleen and $4.8 \%$ for the liver. For all the inserts the ratio for the sphere is larger than for the insert VOI.

\subsection{Activity recovery factors}

Activity recovery factors, which depend on the calibration factor, are shown in Fig. 4. For the liver and spleen the activity recovery is improved when calibration factors are applied which more closely match the patient organ size and shape. See for example the difference between recovery factors for the liver when the sphere and organspecific calibrations are used. Similar activity recovery factors are seen

Table 4

Calibration factors for the patient organ models for images reconstructed with attenuation and scatter correction applied.

\begin{tabular}{lr}
\hline Insert & Calibration factor (cps/ MBq) \\
\hline Liver & $7.54 \pm 0.17$ \\
Spleen (central position) & $7.00 \pm 0.14$ \\
Spleen (anatomical position) & $7.98 \pm 0.18$ \\
Left kidney & $6.43 \pm 0.18$ \\
Right kidney & $6.19 \pm 0.15$ \\
\hline
\end{tabular}




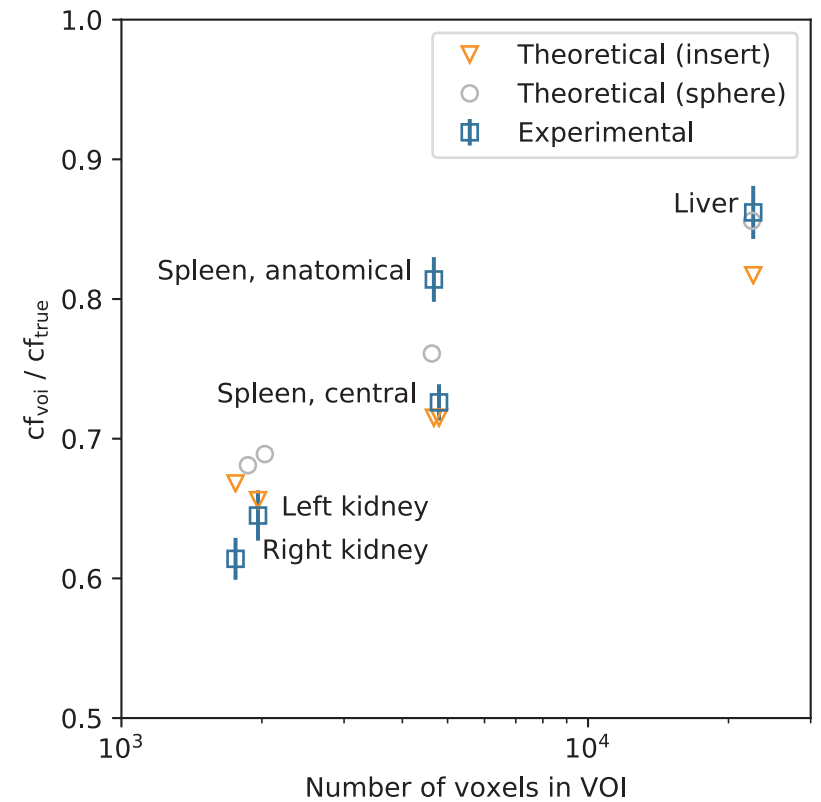

Fig. 3. Ratio of VOI calibration factors $\left(\mathrm{cf}_{\mathrm{voi}}\right)$ to true camera sensitivity $\left(\mathrm{cf}_{\text {true }}\right)$. Data are shown for phantom measurements (blue squares) and corresponding calculated values (orange triangles). Calculations for a sphere of a corresponding volume are also shown (grey circles). (For interpretation of the references to colour in this figure legend, the reader is referred to the web version of this article.)

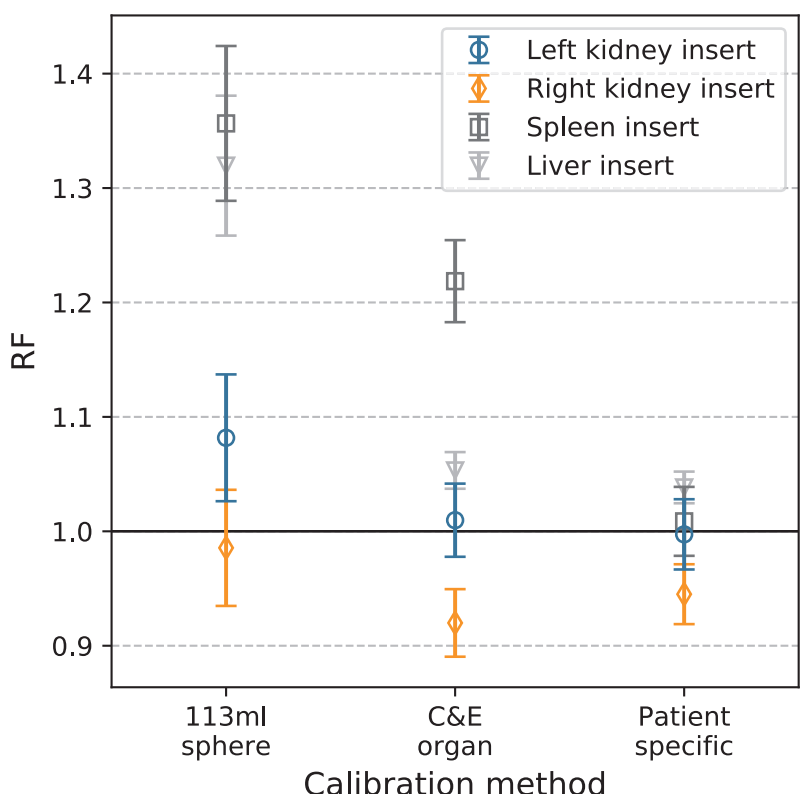

Fig. 4. The recovery factors for the patient inserts with activities quantified using different calibration factors. The line at 1.0 indicates $100 \%$ activity recovery. For the liver and the spleen inserts there is a clear improvement in recovery factor when the organ specific and patient-specific calibration factors are used as opposed to the sphere. It should be noted that for the sphere and C\& E measurements the inserts were located centrally.

for the kidneys with spherical or C\&E kidney calibration factors, as reported in [11].

Activity recovery factors for calibration factors calculated using different spleen positions are compared in Table 5. A $14 \%$ change in recovered activity in the spleen is observed from the central to anatomical position.
Table 5

Comparison of the recovery factors for the patient spleen calculated using calibration factors based on scans of the spleen at the centre of the phantom and in the peripheral anatomical position.

\begin{tabular}{lcc}
\hline Central & Anatomical & Percentage difference (\%) \\
\hline $1.15 \pm 0.03$ & $1.01 \pm 0.03$ & $14 \pm 5$ \\
\hline
\end{tabular}

\subsection{Monte Carlo dose calculations}

\subsubsection{Comparison of voxelised MC simulations of the C\&E organ models to} OLINDA/EXM calculations

The Monte Carlo simulation used to calculate the patient specific doses was validated by comparison of a simulation of voxelised versions of the C\&E organ models and the output of OLINDA/EXM. This compares the output of the simulation to a well established dosimetry standard. The volumes of the voxelised C\&E organ models are all within $1 \%$ of the volumes of the mathematically defined C\&E organs as defined in [18]. The uncertainties on the doses from OLINDA/EXM are approximately $5 \%$ [20]. The statistical uncertainty on the output of the simulation is small for the gamma doses and the beta self-doses, $\sim 0.02 \%$ and $\sim 0.008 \%$ respectively. The uncertainty is larger for the beta cross-doses, $\sim 1 \%-\sim 7 \%$, as proportionally fewer beta particles will travel far enough to reach the other organs causing an increase in the statistical uncertainty.

The doses calculated using the voxelized MC simulation are consistent with OLINDA/EXM to within 5\%. The agreement between this simulation and OLINDA/EXM is consistent with other comparisons between different Monte Carlo codes for dosimetry [21-23].

\subsubsection{Patient-specific dose calculations}

Doses based on experimental data, quantified with a range of calibration factors, calculated using OLINDA/EXM and the patient-specific Monte Carlo simulation are shown in Table 6. The dominant contribution to the dose to each organ is the self-dose, the cross dose being approximately 1000 times smaller. The dominant component of the total dose is from beta particles. Gamma rays contribute between 2 and $5 \%$ of the dose from beta particles. The beta component of cross-dose is negligible for ${ }^{177} \mathrm{Lu}$ (three orders of magnitude lower than the gamma component) and is not reported by OLINDA/EXM in these cases. The self-dose is dominated by the dose from beta particles, which give 96 to $98 \%$ of the self-dose to these organs. The agreement between the patient-specific MC calculations of the gamma component of the self-dose and that reported by OLINDA/EXM is between $99.99 \%$ and $94.3 \%$. The uncertainty on the MC dose calculations is dominated by the uncertainty on the activity in each insert (approximately $2 \%$ ). The activity recovered using the calibration factor for the spleen in the anatomical position is used for the patient-specific calibration of the spleen insert. For organ self-dose, the dominant contribution, calculations with OLINDA/EXM using a patient-specific calibration factor agree with the MC calculated values to within 5\%. OLINDA/EXM consistently underestimates the cross-dose for all source-target pairs apart from the spleen-to-kidney. This is due to the differences in volume between the patient organs and the models used to calculate the S-factors in OLINDA/EXM. The self-dose dominates the cross-dose so the differences in cross-dose do not have a significant impact on the total dose.

\section{Discussion}

\subsection{Patient-specific activity quantification}

There is a clear variation in calibration factors across different patient- specific organ inserts, see Table 4. From the table, it can be seen that there is a significant difference between the calibration factors for the spleen and liver and those for the kidneys. There is also a significant 
Table 6

Total absorbed dose from ${ }^{177} \mathrm{Lu}$, calculated using the patient specific MC simulation and OLINDA/EXM with different activity calibration factors.

\begin{tabular}{|c|c|c|c|c|c|}
\hline \multirow[t]{2}{*}{ Organ } & \multicolumn{5}{|c|}{ Dose (mGy/MBq) } \\
\hline & Sphere $\mathrm{cf}$ & C\&E cf & Patient cf & Administered activity & MC simulation \\
\hline Liver & $6.9 \pm 0.5$ & $5.51 \pm 0.31$ & $5.43 \pm 0.32$ & $5.23 \pm 0.28$ & $5.11 \pm 0.04$ \\
\hline Spleen & $11.6 \pm 0.8$ & $10.4 \pm 0.7$ & $8.6 \pm 0.6$ & $8.5 \pm 0.5$ & $8.25 \pm 0.12$ \\
\hline Kidneys & $22.0 \pm 1.5$ & $20.6 \pm 1.3$ & $20.7 \pm 1.3$ & $21.3 \pm 1.3$ & $20.64 \pm 0.24$ \\
\hline Left kidney & NR & NR & NR & NR & $20.54 \pm 0.31$ \\
\hline Right kidney & NR & NR & NR & NR & $20.8 \pm 0.4$ \\
\hline
\end{tabular}

difference between the calibration factors for the spleen located in a central position and in the anatomical position. The theoretical ratios between $\mathrm{cf}_{\mathrm{voi}}$ and $\mathrm{cf}_{\text {true }}$, shown in Fig. 3, are in agreement with the experimental ratios (within three standard deviations). This agreement, and the clear difference between theoretical ratios for the insert VOIs and spheres of the same volume, confirm the dependence on shape of the calibration factor for patient-specific geometries. Each insert VOI will sample a different region of the spatially varying SPECT pointspread-function (PSF). As such the FWHM used for the theoretical calculations represents an average value for the SPECT field-of-view. The agreement could be therefore be further improved by accounting for this spatial variation in the theoretical calculation. To account for the increased difference between the experimental and theoretical values for the offset spleen the performance of attenuation correction across the field of view must also be accommodated in the imaging corrections.

The shape and volume dependence of activity calibration is also reflected in the activity recovery factors presented in Fig. 4. The data presented in this figure give activity recovery factors for organ inserts in the fully assembled phantom, calculated using calibration factors for a $113 \mathrm{ml}$ sphere, an organ-specific model and the patient-specific model. The activity recovery factors for the left and right kidney inserts (blue circle and orange diamond) agree within the measurement uncertainties for all calibration factors. This highlights the similarity in calibration of the two patient organs to the C\&E organ and a sphere of similar size. For this patient phantom the kidneys are positioned close to the centre. For smaller patients the kidneys will be closer to the edge which must be accounted for during calibration. The recovery factors for the liver (light grey triangle) show a significant overestimation of activity in the insert when the sphere calibration factor is used. The close agreement for both the C\&E and patient liver calibrations (within $10 \%$ ) demonstrates the need for consideration of partial volume effects even for larger organs.

For the off-centre spleen insert (dark grey square), only the patientspecific calibration gives a recovery factor within $10 \%$ of the true activity. Table 5 allows the contributions of insert shape and position to the quantification to be isolated. The decrease in recovery factor when the patient spleen is moved from the centre to the anatomical position is $14 \%$. Applying a similar adjustment for the C\&E spleen suggests that the recovery factor for the C\&E spleen at the edge will be approximately 1.06 , closer to the anatomical factor. There will then be an additional improvement of approximately $5 \%$ by using the correct organ shape. This demonstrates the importance of considering spatial, anatomical, position when calculating calibration factors. Further research is required to fully examine both the impact of the position of organs in the body on activity quantification and variation across a wider range of patient anatomies.

\subsection{Absorbed dose calculations}

The average range of beta particles emitted from ${ }^{177} \mathrm{Lu}$ in soft tissue is approximately $2 \mathrm{~mm}$ and few beta particles escape the organ they are emitted in. The shape of the organs therefore does not markedly impact the amount of energy the beta particles deposit in each organ. The consistency between the calculations done using OLINDA/EXM with mass adjustment and the patient-specific MC simulation is due to the small impact of the organ shape. Table 6 shows that, for the total dose to each organ, the agreement between OLINDA/EXM and the patientspecific MC simulation is good if a patient-specific calibration is used. This is to be expected as the total dose to each organ is dominated by the beta component of the self-dose. The differences for the cross-doses indicate the differences between scaling the mass of the C\&E phantoms and using the true volume. This will be significant for isotopes for which a significant fraction of the total dose is due to gamma rays.

\subsection{Application to clinical data}

This work has demonstrated the importance of patient and organ specific calibration of SPECT images for accurate patient dosimetry in MRT. Such calibration is essential for the accurate quantification of the whole activity distribution represented in these images. More generic calibration using, for example, a small sphere is not adequate when imaging large, non-spherical organs. Calibration assuming that the activity in both kidneys is divided equally may also be unsuitable.

The difference in calibration factor for the 3D printed C\&E printed model and the patient liver is $1.5 \%$, while the difference in volume is $19 \%$. When considering the $80 \%$ range of liver masses for our patient's age range ( $1200 \mathrm{~g}$ to $1820 \mathrm{~g}$ with a median mass of $1460 \mathrm{~g}$ ), a larger difference of $41 \%$ [24] is observed. For the spleen the difference in calibration factor when using the C\&E model and patient spleen is $19 \%$ and the difference in volume is $67 \%$. The $80 \%$ range of spleen masses is $85 \mathrm{~g}$ to $258 \mathrm{~g}$ with a median of $162 \mathrm{~g}$, a difference of $101 \%$. The mass of the patient spleen is approximately 1.5 times the upper bound of the $80 \%$ range [24]. In both cases the differences in mass between the CATIE organs and the C\&E models is less than the difference in masses between the extremes of the $80 \%$ range of organ masses. It is therefore expected that greater deviations in calibration factor would be observed for other patients. In the case of the spleen the variation in calibration factor is also affected by the position, which must also be accounted for.

\section{Conclusion}

Accurate activity quantification is vital for accurate, patient-specific dosimetry to be performed. C\&E calibration factors are suitable for patients presenting with organs similar in size, shape and position to $C \&$ E defined organs. When used in patients with asymmetric kidney function, the OLINDA/EXM assumption of the kidneys as a single organ fails to account for this. Individual kidney doses, taking into account cross-doses from adjacent sources, are required for accurate dosimetry in this configuration. The C\&E S-factors in OLINDA/EXM are suitable for cases where the patient radiation dose is dominated by the contribution of beta particles if the masses of the C\&E organs are scaled to those of the patient, provided the correct activity is used. If the crossdose is a significant source of dose then patient-specific S-factors are required.

The results presented in this work demonstrate that organ specific calibration is necessary for accurate activity quantification in clinical SPECT scans. This calibration must account for the size, shape and 
position of the patient organ. Corrections applied during reconstruction of the image are not sufficient to account for these effects for this patient. The patient-specific calibration presented in this work confirms the benefit of using more representative non-spherical calibration factors. The ready availability of C\&E based inserts [12] now allows clinical centres to access improved SPECT quantification, with the prospect of further improvements with the use of patient-specific calibrations. We have demonstrated the causes of reduced accuracy for dosimetry in molecular radiotherapy and shown that it is feasible to improve this using anthropomorphic calibration.

\section{Funding}

This work was supported by the Science and Technology Facilities Council [Grant Nos. ST/M002918/1, ST/I006188/1] and HERMES Medical Solutions Ltd.

\section{References}

[1] Garkavij M, Nickel M, Sjogreen-Gleisner K, Ljungberg M, Ohlsson T, Wingardh K, et al. 177Lu-[DOTA0, Tyr3] octreotate therapy in patients with disseminated neuroendocrine tumors: analysis of dosimetry with impact on future therapeutic strategy. Cancer 2010;115(S4):1084-92. https://doi.org/10.1002/cncr.24796.

[2] Bardies M, Flux G, Lassmann M, Monsieurs M, Savolainen S, Strand SE. Quantitative imaging for clinical dosimetry. Nucl Instr Meth Phys Res A 2006;569(2):467-71. https://doi.org/10.1016/j.nima.2006.08.068. URL: http://linkinghub.elsevier. $\mathrm{com} / \mathrm{retrieve/pii/S016890020601504X.}$

[3] Strigari L, Konijnenberg M, Chiesa C, Bardies M, Du Y, Gleisner K, et al. The evidence base for the use of internal dosimetry in the clinical practice of molecular radiotherapy. Eur J Nucl Med Mol Imaging 2014;41(10):1976-88. https://doi.org/ 10.1007/s00259-014-2824-5. URL: http://www.ncbi.nlm.nih.gov/pubmed/ 24915892.

[4] Stabin M, Sparks R, Crowe E. OLINDA/EXM: the second-generation personal computer software for internal dose assessment in nuclear medicine. J Nucl Med 2005;46(6):1023-7. URL: http://jnm.snmjournals.org/content/46/6/1023. abstract.arXiv: http://jnm.snmjournals.org/content/46/6/1023.full.pdf + html.

[5] Stabin M, Farmer A. Olinda/exm 2.0: The new generation dosimetry modeling code. J Nucl Med 2012;53(supplement 1):585. URL http://jnm.snmjournals.org/ content/53/supplement 1/585.abstract.

[6] Dewaraja Y, Frey E, Sgouros G, Brill A, Roberson P, Zanzonico P, et al. MIRD pamphlet No. 23: quantitative SPECT for patient-specific 3-dimensional dosimetry in internal radionuclide therapy. J Nucl Med 2012;53(8):1310-25. https://doi.org/ 10.2967/jnumed.111.100123.

[7] Willowson K, Tapner M, Bailey D. A multicentre comparison of quantitative 90Y PET/CT for dosimetric purposes after radioembolization with resin microspheres: the QUEST Phantom Study. Eur J Nucl Med Mol Imaging 2015;42(8):1202-22. https://doi.org/10.1007/s00259-015-3059-9.

[8] Hutton B, Buvat I, Beekman F. Review and current status of SPECT scatter correction. Phys Med Biol 2011;56(14):R85-112. https://doi.org/10.1088/0031-9155/ 56/14/R01. URL: http://www.ncbi.nlm.nih.gov/pubmed/21701055.
[9] Gear J, Long C, Rushforth D, Chittenden S, Cummings C, Flux G. Development of patient-specific molecular imaging phantoms using a 3D printer. Med Phys 2014;41(8part1):082502. https://doi.org/10.1118/1.4887854.

[10] Gear J, Cummings C, Craig A, Divoli A, Long C, Tapner M, et al. Abdo-Man: a 3Dprinted anthropomorphic phantom for validating quantitative SIRT. EJNMMI Phys 2016;3(1):17. https://doi.org/10.1186/s40658-016-0151-6. URL:http:// ejnmmiphys.springeropen.com/articles/10.1186/s40658-016-0151-6.

[11] Tran-Gia J, Schlogl S, Lassmann M. Design and fabrication of kidney phantoms for internal radiation dosimetry using 3d printing technology. J Nucl Med 2016;57(12):1998-2005. https://doi.org/10.2967/jnumed.116.178046.

[12] Robinson A, Tipping J, Cullen D, Hamilton D, Brown R, Flynn A, et al. Organspecific SPECT activity calibration using 3d printed phantoms for molecular radiotherapy dosimetry. EJNMMI Phys 2016;3(12). https://doi.org/10.1186/ s40658-016-0148-1. URL:http://www.ncbi.nlm.nih.gov/pmc/articles/ PMC4943909/.

[13] Ljungberg M, Celler A, Konijnenberg M, Eckerman K, Dewaraja Y, Sjogreen-Gleisner KMIRD. MIRD pamphlet No. 26: joint EANM/MIRD guidelines for quantitative ${ }^{177} \mathrm{Lu}$ SPECT applied for dosimetry of radiopharmaceutical therapy therapy. J Nucl Med 2016;57(1):151-62. https://doi.org/10.2967/jnumed.115.159012.

[14] He B, Frey E. The impact of 3d volume of interest definition on accuracy and precision of activity estimation in quantitative spect and planar processing methods. Phys Med Biol 2010;55(12)::3535. URL: http://stacks.iop.org/0031 $9155 / 55 / \mathrm{i}=12 / \mathrm{a}=017$

[15] Bolch W, Eckerman K, Sgouros G, Thomas S. MIRD pamphlet no. 21: a generalized schema for radiopharmaceutical dosimetry - standardization of nomenclature. J Nucl Med 2009;50(3):477-84.

[16] Santin G, Strul D, Lazaro D, Simon L, Krieguer M, Vieira Martins M, et al. Gate: a geant4-based simulation platform for pet and spect integrating movement and time management. IEEE Trans Nucl Sci 2003;50(5):1516-21. https://doi.org/10.1109/ TNS.2003.817974.

[17] Agostinelli S, Allison J, Amako K, Apostolakis J, Araujo H, Arce P, et al. Geant4 - a simulation toolkit. Nucl Instr Meth Phys Res A 2003;506(3):250-303. URL: http:// www.sciencedirect.com/science/article/pii/S0168900203013688.

[18] Cristy M, Eckerman K. Specific absorbed fractions of energy at various ages from internal photon sources. Oak Ridge National Laboratory; 1987.

[19] Wevrett J, Fenwick A, Scuffham J, Nisbet A. Development of a calibration protocol for quantitative imaging for molecular radiotherapy dosimetry. Radiat Phys Chem 2017;140:355-60. https://doi.org/10.1016/j.radphyschem.2017.02.053.

[20] Stabin M. Uncertainties in internal dose calculations for radiopharmaceuticals. J Nucl Med 2008;49(5):853-60. https://doi.org/10.2967/jnumed.107.048132.

[21] Villoing D, Marcatili S, Garcia MP, Bardies M. Internal dosimetry with the Monte Carlo code GATE: validation using the ICRP/ICRU female reference computational model. Phys Med Biol 2017;62(5):1885-904. https://doi.org/10.1088/1361-6560/ 62/5/1885. URL: http://iopscience.iop.org/0031-9155/62/5/1885\%5Cnhttp:// stacks.iop.org $/ 0031-9155 / 62 / \mathrm{i}=5 / \mathrm{a}=1885$ ? $\mathrm{key}=$ crossref 2e0a515749981ef6189deeb002051ab4.

[22] Pacilio M, Lanconelli N, Lo Meo S, Betti M, Montani L, Torres Aroche L, et al. Differences among Monte Carlo codes in the calculations of voxel S values for radionuclide targeted therapy and analysis of their impact on absorbed dose evaluations. Med Phys 2009;36(5):1543-52. https://doi.org/10.1118/1.3103401.

[23] Parach A, Rajabi H. A comparison between GATE4 results and MCNP4B published data for internal radiation dosimetry. Nuklearmedizin 2011;50(3):122-33. https:// doi.org/10.3413/Nukmed-0363-10-10.

[24] Report of the task group on reference man. icrp publication 23. Tech. Rep.; ICRP; 1975. 